\title{
Bounded solutions for fuzzy integral equations of fractional order
}

Haihua Wang*

\section{"Correspondence:}

wanghoiwan@163.com

Department of Mathematics, Hunan

University of Science and

Technology, Xiangtan, People's

Republic of China

\begin{abstract}
In this paper, we consider sufficient conditions for the boundedness of every solution of fractional order fuzzy integral equations. Some examples are given to illustrate our results.
\end{abstract}

MSC: $34 \mathrm{C} 11 ; 26 \mathrm{E} 50 ; 45 \mathrm{G} 10$

Keywords: boundedness; fractional order; generalized Gronwall inequality

\section{Introduction}

The concept of fuzzy derivative was first introduced by Chang and Zadeh [1]. Kaleva [2], Puri and Ralescu [3] introduced the notion of fuzzy derivative as an extension of the Hukuhara derivative and the fuzzy integral, which was the same as that proposed by Dubois and Prade [4]. There has been a significant development in the study of fuzzy differential and integral equations (see, for example, [5-8], and the references therein). Under suitable conditions, it was proved in [9] that the boundedness of solutions of the following fuzzy integral equation:

$$
x(t)=\int_{0}^{t} G(t, s) x(s) d s+f(t)
$$

where

$$
G: \Delta:=\{(t, s): 0 \leq s \leq t<\infty\} \rightarrow \mathbb{R},
$$

is continuous and $f:[0,+\infty) \rightarrow E^{n}$ is bounded. Furthermore, authors considered the boundedness of solutions of the fuzzy differential equation

$$
x^{\prime}(t)=f(t, x(t)), \quad t \in \mathbb{R}^{+}=[0,+\infty),
$$

where $f: \mathbb{R}^{+} \times E^{n} \rightarrow E^{n}$ is continuous.

The purpose of this paper is to investigate the boundedness of solutions of the following fuzzy functional integral equation with fractional order:

$$
x(t)=\int_{0}^{t}(t-s)^{q-1}\left[G_{1}(t, s) x(s)+G_{2}(t, s) x(\theta(s))\right] d s+f(t),
$$

(c) The Author(s) 2018. This article is distributed under the terms of the Creative Commons Attribution 4.0 International License (http://creativecommons.org/licenses/by/4.0/), which permits unrestricted use, distribution, and reproduction in any medium, provided you give appropriate credit to the original author(s) and the source, provide a link to the Creative Commons license, and indicate if changes were made. 
where

$$
G_{i}: \Delta:=\{(t, s): 0 \leq s \leq t<\infty\} \rightarrow \mathbb{R}
$$

are continuous for $i=1,2,0<q \leq 1, f \in C\left(\mathbb{R}^{+}, E^{n}\right), 0 \leq \theta(t) \leq t$.

Moreover, we also study the boundedness of solutions of the fuzzy integral equation

$$
x(t)=\int_{0}^{t}(t-s)^{q-1} G(t, s) x(s) d s+f(t)
$$

where $G \in C(\Delta, \mathbb{R})$ and $f \in C_{1-q}\left(\mathbb{R}^{+}, E^{n}\right)$,

$$
C_{1-q}\left(\mathbb{R}^{+}, E^{n}\right)=\left\{x \in C\left((0,+\infty), E^{n}\right): t^{1-q} x(t) \in C\left(\mathbb{R}^{+}, E^{n}\right)\right\} .
$$

Inspired by the work of $[9,10]$, in the present paper we aim to establish some sufficient conditions for the boundedness of every solution of fractional order fuzzy integral equations as well as certain fuzzy differential equations. The paper is organized as follows. In Section 2, we present some preliminaries and lemmas. We also correct and complete some previous results. In Section 3, we discuss the boundedness of solution for a particular fuzzy fractional differential equation. In Section 4, the boundedness of solutions for problems (1.3) and (1.4) are given, respectively.

\section{Preliminaries and lemmas}

Let $P_{k}\left(\mathbb{R}^{n}\right)$ be the family of all nonempty compact convex subsets of $\mathbb{R}^{n}$. For $A, B \in P_{k}\left(\mathbb{R}^{n}\right)$, the Hausdorff-Pompeiu metric is defined by

$$
d_{H}(A, B)=\max \left\{\sup _{a \in A} \inf _{b \in B}\|a-b\|, \sup _{b \in B} \inf _{a \in A}\|a-b\|\right\} .
$$

A fuzzy set in $\mathbb{R}^{n}$ is a function with domain $\mathbb{R}^{n}$ and values in $[0,1]$, that is, an element of $[0,1]^{\mathbb{R}^{n}}$.

We denote by $E^{n}$ the space of all fuzzy sets $u: \mathbb{R}^{n} \rightarrow[0,1]$ with the following properties:

(i) $u$ is normal, that is, there exists $x_{0} \in \mathbb{R}^{n}$ such that $u\left(x_{0}\right)=1$;

(ii) $u$ is fuzzy convex;

(iii) $u$ is upper-semicontinuous;

(iv) $[u]^{0}=\operatorname{cl}\left\{x \in \mathbb{R}^{n} \mid u(x)>0\right\}$ is compact.

$E^{1}$ is called the space of fuzzy numbers. Obviously $\mathbb{R} \subset E^{1}$. Here $\mathbb{R} \subset E^{1}$ is understood as $\mathbb{R}=\left\{\chi_{\{x\}}: x\right.$ is the usual real number $\}$.

Let $u \in E^{n}$, then the set

$$
[u]^{\alpha}=\left\{x \in \mathbb{R}^{n}: u(x) \geq \alpha\right\}, \quad \alpha \in(0,1]
$$

is called the $\alpha$-level set of $u$.

The fuzzy zero is defined by

$$
\chi_{\{0\}}(x)= \begin{cases}0, & x \neq 0 \\ 1, & x=0\end{cases}
$$


Let $d_{\infty}: E^{n} \times E^{n} \rightarrow[0,+\infty)$ be defined by

$$
d_{\infty}(u, v)=\sup \left\{d_{H}\left([u]^{\alpha},[v]^{\alpha}\right): \alpha \in[0,1]\right\}
$$

where $d_{H}$ is the Hausdorff-Pompeiu metric for nonempty compact convex subsets of $\mathbb{R}^{n}$. Then $\left(E^{n}, d_{\infty}\right)$ is a complete metric space. This defines a linear structure on $E^{n}$ such that, for all $u, v, w, z \in E^{n}$ and $\lambda \in \mathbb{R}$, we have $d_{\infty}(u+w, v+w)=d_{\infty}(u, v)$ and $d_{\infty}(\lambda u, \lambda v)=$ $|\lambda| d_{\infty}(u, v)$. Also, we can prove that $d_{\infty}(u+v, w+z) \leq d_{\infty}(u, w)+d_{\infty}(v, z)$.

A mapping $u: T \rightarrow E^{n}$ is bounded, where $T$ is an interval of the real line, if there exists $r>0$ such that

$$
d_{\infty}\left(u(x), \chi_{\{0\}}\right) \leq r, \quad \forall x \in T .
$$

Let $u, v \in E^{n}$ and $c$ be a positive number, the addition $u+v$ and $c v$ in $E^{n}$ are defined in terms of the $\alpha$-level sets by

$$
[u+v]^{\alpha}=[u]^{\alpha}+[v]^{\alpha}, \quad[c v]^{\alpha}=c[v]^{\alpha}
$$

for every $\alpha \in[0,1]$.

A fuzzy function $f: T \rightarrow E^{n}$ is measurable if, for all $\alpha \in[0,1]$, the set-valued mapping $f_{\alpha}: T \rightarrow P_{k}\left(\mathbb{R}^{n}\right)$ defined by $f_{\alpha}(t)=[f(t)]^{\alpha}$ is measurable.

We denote by $S_{f}^{1}$ the set of all Lebesgue integrable section of $F: T \rightarrow P_{k}\left(\mathbb{R}^{n}\right)$, that is, $S_{f}^{1}=\left\{g \in L^{1}\left(T, \mathbb{R}^{n}\right): g(t) \in f(t)\right.$ a.e. $\}$.

A fuzzy function $f: T \rightarrow E^{n}$ is integrably bounded if there exists an integrable function $h$ such that $\|x\| \leq h(t)$ for all $x \in f_{0}(t)$. A measurable and integrably bounded fuzzy function $f: T \rightarrow E^{n}$ is said to be integrable over $T$ if there exists $F \in E^{n}$ such that $F_{\alpha}=\int_{T} f_{\alpha}(t) d t=$ $\left\{\int_{T} g(t) d t: g \in S_{f_{\alpha}}^{1}\right\}$ for all $\alpha \in[0,1]$.

Definition 2.1 ([11]) The Riemann-Liouville fractional integral of order $\beta>0$ for a function $f: \mathbb{R}^{+} \rightarrow \mathbb{R}$ is given by

$$
I^{\beta} f(t)=\frac{1}{\Gamma(\beta)} \int_{0}^{t}(t-s)^{\beta-1} f(s) d s .
$$

Definition 2.2 ([11]) The Riemann-Liouville fractional derivative of order $0<\beta<1$ for a function $f: \mathbb{R}^{+} \rightarrow \mathbb{R}$ is defined by

$$
D^{\beta} f(t)=\frac{1}{\Gamma(1-\beta)} \frac{d}{d t} \int_{0}^{t}(t-s)^{-\beta} f(s) d s
$$

Definition 2.3 ([12]) Let $f: \mathbb{R}^{+} \rightarrow E^{n}$ be an integrable fuzzy function. The fuzzy fractional integral of order $\beta>0$ of the function $f$,

$$
I^{\beta} f(t)=\frac{1}{\Gamma(\beta)} \int_{0}^{t}(t-s)^{\beta-1} f(s) d s,
$$

is defined by

$$
I^{\beta} f(t)(x)=\sup \left\{\alpha \in[0,1]: x \in I^{\beta} f_{\alpha}\right\} .
$$


Its level sets are given by

$$
\left[I^{\beta} f(t)\right]^{\alpha}=\left\{x \in \mathbb{R}^{n}: I^{\beta} f(t)(x) \geq \alpha\right\}, \quad \alpha \in[0,1]
$$

that is, we have

$$
\left[I^{\beta} f(t)\right]^{\alpha}=\frac{1}{\Gamma(\beta)} \int_{0}^{t}(t-s)^{\beta-1}[f(s)]^{\alpha} d s .
$$

Let $x, y \in E^{n}$. If there exists $z \in E^{n}$ such that $x=y+z$, then we call $z$ the $H$-difference of $x$ and $y$, denoted by $x-H y$.

A fuzzy function $f: T \rightarrow E^{n}$ is said to be differentiable at $t \in T$ if there exists $\frac{d}{d t} f(t) \in E^{n}$ such that the limits

$$
\lim _{h \rightarrow 0+} \frac{f(t+h)-{ }_{H} f(t)}{h} \text { and } \lim _{h \rightarrow 0+} \frac{f(t)-H f(t-h)}{h}
$$

exist and are equal to $\frac{d}{d t} f(t)$.

Definition 2.4 ([12]) If $f:[0,+\infty) \rightarrow E^{n}$, then the Riemann-Liouville fractional derivative of order $0<\beta \leq 1$ of $f$ is defined as

$$
D^{\beta} f(t)=\frac{1}{\Gamma(1-\beta)} \frac{d}{d t} \int_{0}^{t}(t-s)^{-\beta} f(s) d s
$$

provided that the equation defines a fuzzy number $D^{\beta} f(t) \in E^{n}$. It is easy to see that $D^{\beta} f(t)=\frac{d}{d t} I^{1-\beta} f(t), t \in[0,+\infty)$.

Definition 2.5 ([11]) For $\alpha>0$, the classical Mittag-Leffler function $E_{\alpha}(z)$ and the generalized Mittag-Leffler function $E_{\alpha, \beta}(z)$ are defined by

$$
E_{\alpha}(z)=\sum_{k=0}^{\infty} \frac{z^{k}}{\Gamma(\alpha k+1)}, \quad E_{\alpha, \beta}(z)=\sum_{k=0}^{\infty} \frac{z^{k}}{\Gamma(\alpha k+\beta)} .
$$

Definition 2.6 ([13]) $f: \mathbb{R} \rightarrow \mathbb{R}$ is locally Hölder continuous in $t_{1}$ if there exist a neighborhood $U$ of $t_{1}$ and constants $c>0,0<v<1$ such that, for all $t, s \in U$,

$$
|f(t)-f(s)| \leq c|t-s|^{\nu} .
$$

Lemma 2.1 ([14]) Let $m: \mathbb{R}^{+} \rightarrow \mathbb{R}$ be locally Hölder continuous with exponent $\lambda>q$ such that, for any $t_{1}>0$, we have

$$
m\left(t_{1}\right)=0 \quad \text { and } \quad m(t) \leq 0 \quad \text { for } 0 \leq t \leq t_{1} .
$$

Then it follows that $D^{q} m\left(t_{1}\right) \geq 0$.

We note that Lemma 2.1 is very limited in the sense that the condition imposed on the $m$ is quite restrictive. We shall be concerned with the problem of finding the appropriate modifications that are needed to prove the results when $m$ satisfies a weaker assumption. To illustrate this idea, we shall next give the result which is an improvement of Lemma 2.1. 
Lemma 2.2 Let $m: \mathbb{R}^{+} \rightarrow \mathbb{R}$. If there exists $\delta \geq 0$ such that $t^{\delta} m(t)$ is locally Hölder continuous with exponent $\lambda>q$ such that, for any $t_{1}>0$, (2.1) holds, then it follows that $D^{q} m\left(t_{1}\right) \geq 0$.

Proof The proof is similar to Lemma 2.1, but for the sake of completeness, we give the details of it. Let $H(t)=\int_{0}^{t}(t-s)^{-q} m(s) d s$. Consider, for sufficiently small $h>0$ such that $t_{1}-h>0$,

$$
\begin{aligned}
H\left(t_{1}\right)-H\left(t_{1}-h\right)= & \int_{0}^{t_{1}-h}\left[\left(t_{1}-s\right)^{-q}-\left(t_{1}-h-s\right)^{-q}\right] m(s) d s \\
& +\int_{t_{1}-h}^{t_{1}}\left(t_{1}-s\right)^{-q} m(s) d s=I_{1}+I_{2} .
\end{aligned}
$$

Since $\left[\left(t_{1}-s\right)^{-q}-\left(t_{1}-h-s\right)^{-q}\right]<0$ for $0 \leq s \leq t_{1}-h$ and $m(s) \leq 0$ by hypothesis, we have $I_{1} \geq 0$. Also, $H\left(t_{1}\right)-H\left(t_{1}-h\right) \geq I_{2}$. Since $t^{\delta} m(t)$ is locally Hölder continuous and $m\left(t_{1}\right)=0$, there exists a constant $K\left(t_{1}\right)>0$ such that, for $t_{1}-h \leq s \leq t_{1}+h$,

$$
-K\left(t_{1}\right)\left(t_{1}-s\right)^{\lambda} \leq s^{\delta} m(s) \leq K\left(t_{1}\right)\left(t_{1}-s\right)^{\lambda}
$$

where $\lambda>q$ and $0<\lambda<1$. We then get

$$
\begin{aligned}
I_{2} & =\int_{t_{1}-h}^{t_{1}}\left(t_{1}-s\right)^{-q} s^{-\delta} s^{\delta} m(s) d s \geq-K\left(t_{1}\right) \int_{t_{1}-h}^{t_{1}}\left(t_{1}-s\right)^{\lambda-q} s^{-\delta} d s \\
& \geq \frac{-K\left(t_{1}\right)}{\left(t_{1}-h\right)^{\delta}(\lambda+1-q)} h^{\lambda+1-q} .
\end{aligned}
$$

Hence $H\left(t_{1}\right)-H\left(t_{1}-h\right) \geq-\frac{K\left(t_{1}\right)}{\left(t_{1}-h\right)^{\delta}(\lambda+1-q)} h^{\lambda+1-q}$. Letting $h \rightarrow 0^{+}$, we obtain $H^{\prime}\left(t_{1}\right) \geq 0$, which implies $D^{q} m\left(t_{1}\right)=\frac{1}{\Gamma(1-q)} H^{\prime}\left(t_{1}\right) \geq 0$ and the proof is complete.

Remark 2.1 If there exists $0 \leq \delta<1$ such that $t^{\delta} m(t)$ is locally Hölder continuous with exponent $\lambda$, then for any $c \in \mathbb{R}, t^{\delta}(m(t)-c)$ is also locally Hölder continuous with exponent $\max \{\lambda, \delta\}$.

In Theorem 4.1 of [14], authors considered the global existence of solution of the problem

$$
D^{q} u(t)=g(t, u(t)), \quad \lim _{t \rightarrow 0^{+}} t^{1-q} u(t)=u_{0},
$$

by constructing the auxiliary problem

$$
D^{q} u(t)=g(t, u(t))+\epsilon, \quad \lim _{t \rightarrow 0^{+}} t^{1-q} u(t)=u_{0}+\epsilon, \quad \epsilon>0 .
$$

The idea is very interesting, but we find out that there are no conditions to ensure the existence of solution for $\epsilon>0$. So, we revise the corresponding theorem as follows. Next, we give the notion of maximal solution which is of the same form as that in [15]. 
Definition 2.7 Let $r(t)$ be a solution of the scalar differential equation $(2.2)$ on $(0,+\infty)$. Then $r(t)$ is said to be a maximal solution of (2.2) if, for every solution $u(t)$ of (2.2) existing on $(0,+\infty)$, the inequality $u(t) \leq r(t), t \in(0,+\infty)$ holds.

Theorem 2.1 Let $C_{1-q}\left(\mathbb{R}^{+}, \mathbb{R}\right)=\left\{x \in C((0,+\infty), \mathbb{R}): t^{1-q} x(t) \in C\left(\mathbb{R}^{+}, \mathbb{R}\right)\right\}$. Assume that m$\eta \in C_{1-q}\left(\mathbb{R}^{+}, \mathbb{R}\right)$, there exists $0 \leq \delta<1$ such that $t^{\delta}(m-\eta)$ is locally Hölder continuous with exponent $\lambda>q, g \in C((0,+\infty) \times \mathbb{R}, \mathbb{R})$ satisfies

$$
g(t, x)-g(t, y) \geq \frac{L(x-y)}{t^{q} \Gamma(1-q)}, \quad \text { wherever } t \in(0,+\infty), x \geq y, L>1
$$

and

$$
D^{q} m(t) \leq g(t, m(t)), \quad t \in(0,+\infty)
$$

Let $\eta(t)$ be the maximal solution of $(2.2)$ in $(0,+\infty)$ such that $\lim _{t \rightarrow 0^{+}} t^{1-q} m(t)<u_{0}$, then we have $m(t) \leq \eta(t), t \in(0,+\infty)$.

Proof For $\epsilon>0$, let $\eta_{\epsilon}(t)=\eta(t)-\epsilon, t \in(0,+\infty)$. Then, by Definition 2.2 and (2.3),

$$
\begin{aligned}
D^{q} \eta_{\epsilon}(t) & =D^{q} \eta(t)-\frac{\epsilon}{t^{q} \Gamma(1-q)} \\
& =g\left(t, \eta_{\epsilon}(t)+\epsilon\right)-\frac{\epsilon}{t^{q} \Gamma(1-q)} \\
& \geq g\left(t, \eta_{\epsilon}(t)\right)+\frac{(L-1) \epsilon}{t^{q} \Gamma(1-q)} \\
& >g\left(t, \eta_{\epsilon}(t)\right), \quad t \in(0,+\infty)
\end{aligned}
$$

and $\lim _{t \rightarrow 0^{+}} t^{1-q} \eta_{\epsilon}(t)=u_{0}$. Next, we claim $m(t)-\eta_{\epsilon}(t)<0, t \in(0,+\infty)$. Assume that it is not true. Then, for $\lim _{t \rightarrow 0^{+}} t^{1-q}\left(m(t)-\eta_{\epsilon}(t)\right)<0$, it follows that there exists $t_{1} \in(0,+\infty)$ such that $m\left(t_{1}\right)-\eta_{\epsilon}\left(t_{1}\right)=0$ and $m(t)-\eta_{\epsilon}(t)<0, t \in\left(0, t_{1}\right)$. Using Lemma 2.2 and Remark 2.1, we obtain $D^{q} m\left(t_{1}\right) \geq D^{q} \eta_{\epsilon}\left(t_{1}\right)$. So, we have

$$
g\left(t_{1}, m\left(t_{1}\right)\right) \geq D^{q} m\left(t_{1}\right) \geq D^{q} \eta_{\epsilon}\left(t_{1}\right)>g\left(t_{1}, \eta_{\epsilon}\left(t_{1}\right)\right),
$$

which contradicts with $m\left(t_{1}\right)=\eta_{\epsilon}\left(t_{1}\right)$. That is, $m(t)<\eta_{\epsilon}(t), t \in(0,+\infty)$. By the arbitrariness of $\epsilon$, we can get $m(t) \leq \eta(t), t \in(0,+\infty)$.

Corollary 2.1 Let $g \in C((0,+\infty) \times \mathbb{R}, \mathbb{R})$ satisfy (2.3). Let $v, \eta \in C_{1-q}\left(\mathbb{R}^{+}, \mathbb{R}\right)$, and there exists $1-q \leq \delta<1$ such that $t^{\delta}\left[I^{q} g(t, v(t))-\eta\right]$ is locally Hölder continuous with exponent $\lambda>q$. Moreover, $\eta(t)$ is the maximal solution of $(2.2)$ existing in $(0,+\infty)$. If

$$
v(t) \leq t^{q-1} v_{0}+\int_{0}^{t}(t-s)^{q-1} g(s, v(s)) d s, \quad t \in(0,+\infty)
$$

where $v_{0}<u_{0}$, then $v(t) \leq \eta(t)$ holds in $(0,+\infty)$. 
Proof Let $V(t)$ be the right-hand side of (2.5), so that $v(t) \leq V(t)$ and $D^{q} V(t)=g(t, v(t))$, $\lim _{t \rightarrow 0^{+}} t^{1-q} V(t)=v_{0}<u_{0}$. By $(2.3), D^{q} V(t) \leq g(t, V(t))$. Hence Theorem 2.1 implies that $V(t) \leq \eta(t)$ in $(0,+\infty)$, thus $v(t) \leq \eta(t)$ holds.

Theorem 2.2 Let $g:(0,+\infty) \times \mathbb{R} \rightarrow \mathbb{R}$

$$
(t, u) \rightarrow g(t, u)
$$

be continuous and satisfy inequality (2.3), and let $\eta$ be the maximal solution of (2.2) existing for $t \in(0,+\infty)$. Let $f:(0,+\infty) \times E^{n} \rightarrow E^{n}$ be continuous such that

$$
d_{\infty}\left(f(s, x), \chi_{\{0\}}\right) \leq g\left(s, d_{\infty}\left(x, \chi_{\{0\}}\right)\right), \quad \forall s \in(0,+\infty), x \in E^{n}
$$

If there exists $1-q \leq \delta<1$ such that $t^{\delta}\left[I^{q} g\left(t, d_{\infty}\left(x(t), \chi_{\{0\}}\right)\right)-\eta\right]$ is locally Hölder continuous with exponent $\lambda>q$ such that

$$
d_{\infty}\left(x_{0}, \chi_{\{0\}}\right)<u_{0}
$$

then all solutions $x$ of

$$
x(t)=t^{q-1} x_{0}+\int_{0}^{t}(t-s)^{q-1} f(s, x(s)) d s
$$

satisfy

$$
d_{\infty}\left(x(t), \chi_{\{0\}}\right) \leq \eta(t), \quad t \in(0,+\infty) .
$$

Moreover, if $\eta$ is bounded, then $x$ is bounded.

Proof Let $m(t)=d_{\infty}\left(x(t), \chi_{\{0\}}\right), t \in(0,+\infty)$. Then

$$
\begin{aligned}
m(t) & =d_{\infty}\left(t^{q-1} x_{0}+\int_{0}^{t}(t-s)^{q-1} f(s, x(s)) d s, \chi_{\{0\}}\right) \\
& \leq t^{q-1} d_{\infty}\left(x_{0}, \chi_{\{0\}}\right)+\int_{0}^{t}(t-s)^{q-1} d_{\infty}\left(f(s, x(s)), \chi_{\{0\}}\right) d s \\
& \leq t^{q-1} d_{\infty}\left(x_{0}, \chi_{\{0\}}\right)+\int_{0}^{t}(t-s)^{q-1} g(s, m(s)) d s, \quad t \in(0,+\infty) .
\end{aligned}
$$

The conclusion is obtained by using Corollary 2.1.

Here, we list some basic properties of class and generalized Mittag-Leffler functions which will be used in the later discussion.

Lemma 2.3 ([16]) Let $0<q<1, \lambda>0$. Then functions $E_{q}$ and $E_{q, q}$ are nonnegative and have the following properties:

(i) For any $t>0, E_{q}\left(-\lambda t^{q}\right) \leq 1, E_{q, q}\left(-\lambda t^{q}\right) \leq \frac{1}{\Gamma(q)}$. Moreover, $E_{q}(0)=1, E_{q, q}(0)=\frac{1}{\Gamma(q)}$.

(ii) For any $t_{1}, t_{2}>0$ and $t_{1} \leq t_{2}, E_{q}\left(-\lambda t_{2}^{q}\right) \leq E_{q}\left(-\lambda t_{1}^{q}\right), E_{q, q}\left(-\lambda t_{2}^{q}\right) \leq E_{q, q}\left(-\lambda t_{1}^{q}\right)$. 
Lemma 2.4 ([16]) Let $q>0$, then the relation between $E_{q}(\cdot)$ and $E_{q, q}(\cdot)$ is given by the following integration:

$$
\int_{0}^{t}(t-s)^{q-1} E_{q, q}\left(-\lambda(t-s)^{q}\right) d s=\frac{1-E_{q}\left(-\lambda t^{q}\right)}{\lambda} .
$$

Lemma 2.5 ([17]) Let $\alpha, \beta, \mu>0$, then the following formulas hold:

$$
\begin{aligned}
& \frac{1}{\Gamma(\mu)} \int_{t}^{x}(x-s)^{\mu-1}(s-t)^{\beta-1} E_{\alpha, \beta}\left(\lambda(s-t)^{\alpha}\right) d s=(x-t)^{\beta+\mu-1} E_{\alpha, \beta+\mu}\left(\lambda(x-t)^{\alpha}\right), \\
& \frac{1}{\Gamma(\mu)} \int_{t}^{x}(s-t)^{\mu-1}(x-s)^{\beta-1} E_{\alpha, \beta}\left(\lambda(x-s)^{\alpha}\right) d s=(x-t)^{\beta+\mu-1} E_{\alpha, \beta+\mu}\left(\lambda(x-t)^{\alpha}\right) .
\end{aligned}
$$

\section{A particular equation}

In this section, firstly, we consider the problem

$$
\left\{\begin{array}{l}
D^{q} x(t)+M x(t)=\sigma(t), \quad t \in(0,+\infty), \\
\lim _{t \rightarrow 0^{+}} t^{1-q} x(t)=x_{0} \in E^{1},
\end{array}\right.
$$

where $0<q \leq 1, M>0$ and $\sigma \in C\left(\mathbb{R}^{+}, E^{1}\right)$. Secondly, we discuss the boundedness of solution for problem (3.1).

Lemma 3.1 ([18]) Let $f: T \rightarrow E^{1}$ be differentiable. Denote $[f(t)]^{\alpha}=\left[f_{\alpha l}(t), f_{\alpha r}(t)\right], \alpha \in[0,1]$. Then $f_{\alpha l}$ and $f_{\alpha r}$ are differentiable and $\left[f^{\prime}(t)\right]^{\alpha}=\left[f_{\alpha l}^{\prime}(t), f_{\alpha r}^{\prime}(t)\right]$.

Lemma 3.2 Let $x:(0,+\infty) \rightarrow E^{1}$. If for each $t \in(0,+\infty)$ there exists $h_{0}>0$ such that the H-differences

$$
x(t+h)-{ }_{H} x(t), \quad \text { and } \quad x(t)-{ }_{H} x(t-h)
$$

exist for all $0<h<h_{0}$, then for each $t \in(0,+\infty)$ and $q>0$, the H-differences

$$
I^{q} x(t+h)-{ }_{H} I^{q} x(t), \quad \text { and } \quad I^{q} x(t)-{ }_{H} I^{q} x(t-h)
$$

also exist for all $0<h<h_{0}$.

Proof We shall prove that the $H$-differences of $I^{q} x(t+h)-{ }_{H} I^{q} x(t)$ exist. Similar reasoning can be used for $I^{q} x(t)-{ }_{H} I^{q} x(t-h)$. First, for $0<h<h_{0}$ and $0 \leq \alpha \leq \beta \leq 1$, it is clear that

$$
\begin{aligned}
& \int_{0}^{h}(t+h-s)^{q-1} x_{\alpha l}(s) d s \leq \int_{0}^{h}(t+h-s)^{q-1} x_{\alpha r}(s) d s, \\
& \int_{0}^{h}(t+h-s)^{q-1} x_{\alpha l}(s) d s \leq \int_{0}^{h}(t+h-s)^{q-1} x_{\beta l}(s) d s
\end{aligned}
$$

and

$$
\int_{0}^{h}(t+h-s)^{q-1} x_{\beta r}(s) d s \leq \int_{0}^{h}(t+h-s)^{q-1} x_{\alpha r}(s) d s .
$$


On the one hand, since the $H$-differences $x(t+h)-{ }_{H} x(t)$ exist, we have

$$
x_{\alpha l}(s+h)-x_{\alpha l}(s) \leq x_{\alpha r}(s+h)-x_{\alpha r}(s) .
$$

Multiply (3.5) by $(t-s)^{q-1}$ and then integrate on $[0, t]$ to obtain

$$
\begin{aligned}
\int_{h}^{t+h}(t+h-s)^{q-1} x_{\alpha l}(s) d s-\int_{0}^{t}(t-s)^{q-1} x_{\alpha l}(s) d s \leq & \int_{h}^{t+h}(t+h-s)^{q-1} x_{\alpha r}(s) d s \\
& -\int_{0}^{t}(t-s)^{q-1} x_{\alpha r}(s) d s
\end{aligned}
$$

together with (3.2), we can see that

$$
I^{q} x_{\alpha l}(t+h)-I^{q} x_{\alpha l}(t) \leq I^{q} x_{\alpha r}(t+h)-I^{q} x_{\alpha r}(t) .
$$

On the other hand, we check that the family of intervals

$$
\left\{\left[I^{q} x_{\alpha l}(t+h)-I^{q} x_{\alpha l}(t), I^{q} x_{\alpha r}(t+h)-I^{q} x_{\alpha r}(t)\right]: \alpha \in[0,1]\right\}
$$

defines a fuzzy interval. Indeed, for $0 \leq \alpha \leq \beta \leq 1$,

$$
x_{\alpha l}(s+h)-x_{\alpha l}(s) \leq x_{\beta l}(s+h)-x_{\beta l}(s), \quad x_{\beta r}(s+h)-x_{\beta r}(s) \leq x_{\alpha r}(s+h)-x_{\alpha r}(s) .
$$

Multiplying both sides of the above two inequalities by $(t-s)^{q-1}$ and integrating on $[0, t]$, together with (3.3), (3.4), we can get

$$
I^{q} x_{\alpha l}(t+h)-I^{q} x_{\alpha l}(t) \leq I^{q} x_{\beta l}(t+h)-I^{q} x_{\beta l}(t)
$$

and

$$
I^{q} x_{\beta r}(t+h)-I^{q} x_{\beta r}(t) \leq I^{q} x_{\alpha r}(t+h)-I^{q} x_{\alpha r}(t) .
$$

Finally, the left continuity of $I^{q} x_{\alpha l}(t+h)-I^{q} x_{\alpha l}(t)$ and $I^{q} x_{\alpha r}(t+h)-I^{q} x_{\alpha r}(t)$ at $(0,1]$ and their right continuity at 0 with respect to $\alpha$ are guaranteed. Given $\epsilon>0$, for fixed $h \in\left(0, h_{0}\right)$, by the right continuity of $x_{\alpha l}(t+h), x_{\alpha r}(t+h), x_{\alpha l}(t+h)-x_{\alpha l}(t)$ and $x_{\alpha r}(t+h)-x_{\alpha r}(t)$ at 0 , the following inequalities are satisfied:

$$
\begin{aligned}
\left|x_{\alpha l}(t+h)-x_{0 l}(t+h)\right| \rightarrow 0, & \left|\left[x_{\alpha l}(t+h)-x_{\alpha l}(t)\right]-\left[x_{0 l}(t+h)-x_{0 l}(t)\right]\right| \rightarrow 0, \\
\left|x_{\alpha r}(t+h)-x_{0 r}(t+h)\right| \rightarrow 0, & \left|\left[x_{\alpha r}(t+h)-x_{\alpha r}(t)\right]-\left[x_{0 r}(t+h)-x_{0 r}(t)\right]\right| \rightarrow 0
\end{aligned}
$$

as $\alpha \rightarrow 0^{+}$. Hence, we have

$$
\begin{aligned}
& \left|\left[I^{q} x_{\alpha l}(t+h)-I^{q} x_{\alpha l}(t)\right]-\left[I^{q} x_{0 l}(t+h)-I^{q} x_{0 l}(t)\right]\right| \\
& \leq \frac{1}{\Gamma(q)} \int_{-h}^{0}(t-s)^{q-1}\left|x_{\alpha l}(s+h)-x_{0 l}(s+h)\right| d s \\
& \quad+\frac{1}{\Gamma(q)} \int_{0}^{t}(t-s)^{q-1}\left|\left[x_{\alpha l}(s+h)-x_{\alpha l}(s)\right]-\left[x_{0 l}(s+h)-x_{0 l}(s)\right]\right| d s \rightarrow 0, \quad \alpha \rightarrow 0^{+},
\end{aligned}
$$


and similarly for $I^{q} x_{\alpha r}(t+h)-I^{q} x_{\alpha r}(t)$, so that $I^{q} x_{\alpha l}(t+h)-I^{q} x_{\alpha l}(t)$ and $I^{q} x_{\alpha r}(t+h)-I^{q} x_{\alpha r}(t)$ are right continuous at 0 , respectively. Following the same steps as above, we can show the left continuity of $I^{q} x_{\alpha l}(t+h)-I^{q} x_{\alpha l}(t)$ and $I^{q} x_{\alpha r}(t+h)-I^{q} x_{\alpha r}(t)$ at $(0,1]$. Therefore, for $t>0$, $I^{q} x(t+h){ }_{H} I^{q} x(t)$ exists for $0<h<h_{0}$ and the proof is complete.

Theorem 3.1 Problem (3.1) has a unique solution in $(0,+\infty)$, given by

$$
x(t)=x_{0} \chi_{\left\{\Gamma(q) t^{q-1} E_{q, q}\left(-M t^{q}\right)\right\}}+\int_{0}^{t} \sigma(s) \chi_{\left\{(t-s)^{q-1} E_{q, q}\left(-M(t-s)^{q}\right)\right\}} d s, \quad t \in(0,+\infty),
$$

iffor each $t \in(0,+\infty)$, there exists $h_{0}>0$ such that the H-differences

$$
x(t+h)-{ }_{H} x(t), \quad \text { and } \quad x(t)-{ }_{H} x(t-h)
$$

exist for all $0<h<h_{0}$.

Proof Taking $[x(t)]^{\alpha}=\left[x_{\alpha l}(t), x_{\alpha r}(t)\right]$, then by Definitions 2.3, 2.4 and Lemma 3.1, problem (3.1) is written level-wise as follows:

$$
\begin{cases}D^{q} x_{\alpha l}(t)+M x_{\alpha l}(t)=\sigma_{\alpha l}(t), & t \in(0,+\infty) \\ D^{q} x_{\alpha r}(t)+M x_{\alpha r}(t)=\sigma_{\alpha r}(t), & t \in(0,+\infty) \\ \lim _{t \rightarrow 0^{+}} t^{1-q} x_{\alpha l}(t)=\left(x_{0}\right)_{\alpha l}, & \lim _{t \rightarrow 0^{+}} t^{1-q} x_{\alpha r}(t)=\left(x_{0}\right)_{\alpha r}\end{cases}
$$

Using Theorem 4.1 in [11] and $\lim _{t \rightarrow 0^{+}} t^{1-q} x_{\alpha l}(t)=\frac{D^{q-1} x_{\alpha l}\left(0^{+}\right)}{\Gamma(q)}$, we get

$$
x_{\alpha l}(t)=\Gamma(q)\left(x_{0}\right)_{\alpha l} t^{q-1} E_{q, q}\left(-M t^{q}\right)+\int_{0}^{t} \sigma_{\alpha l}(s)(t-s)^{q-1} E_{q, q}\left(-M(t-s)^{q}\right) d s,
$$

and analogously for $x_{\alpha r}(t)$, producing (3.6).

Now we study the fractional differentiability of $x$. Let $t \in(0,+\infty)$ and $h>0$, by Lemma 3.2, the $H$-differences of $I^{1-q} x(t+h)-_{H} I^{1-q} x(t)$ and $I^{1-q} x(t){ }_{H} I^{1-q} x(t-h)$ also exist. Then, for every $\alpha \in[0,1]$, with Lemma 2.5 , we have

$$
\begin{aligned}
\left(\frac{I^{1-q} x(t+h)-I_{H} I^{1-q} x(t)}{h}\right)_{\alpha l}= & \left(x_{0}\right)_{\alpha l} \Gamma(q) \frac{E_{q}\left(-M(t+h)^{q}\right)-E_{q}\left(-M t^{q}\right)}{h} \\
& +\int_{0}^{t} \sigma_{\alpha l}(s) \frac{E_{q}\left(-M(t+h-s)^{q}\right)-E_{q}\left(-M(t-s)^{q}\right)}{h} d s \\
& +\int_{t}^{t+h} \sigma_{\alpha l}(s) \frac{E_{q}\left(-M(t+h-s)^{q}\right)}{h} d s
\end{aligned}
$$

and

$$
\begin{aligned}
\left(\frac{I^{1-q} x(t+h)-{ }_{H} I^{1-q} x(t)}{h}\right)_{\alpha r}= & \left(x_{0}\right)_{\alpha r} \Gamma(q) \frac{E_{q}\left(-M(t+h)^{q}\right)-E_{q}\left(-M t^{q}\right)}{h} \\
& +\int_{0}^{t} \sigma_{\alpha r}(s) \frac{E_{q}\left(-M(t+h-s)^{q}\right)-E_{q}\left(-M(t-s)^{q}\right)}{h} d s \\
& +\int_{t}^{t+h} \sigma_{\alpha r}(s) \frac{E_{q}\left(-M(t+h-s)^{q}\right)}{h} d s .
\end{aligned}
$$


The limits of these functions as $h \rightarrow 0^{+}$uniformly in $\alpha$ are, respectively,

$$
z_{\alpha l}(t)=\sigma_{\alpha l}(t)-\Gamma(q) M t^{q-1}\left(x_{0}\right)_{\alpha l} E_{q, q}\left(-M t^{q}\right)-M \int_{0}^{t} \sigma_{\alpha l}(s)(t-s)^{q-1} E_{q, q}\left(-M(t-s)^{q}\right) d s
$$

and

$$
z_{\alpha r}(t)=\sigma_{\alpha r}(t)-\Gamma(q) M t^{q-1}\left(x_{0}\right)_{\alpha r} E_{q, q}\left(-M t^{q}\right)-M \int_{0}^{t} \sigma_{\alpha r}(s)(t-s)^{q-1} E_{q, q}\left(-M(t-s)^{q}\right) d s
$$

since $E_{q}^{\prime}(t)=\frac{E_{q, q}(t)}{q},\left(x_{0}\right)_{\alpha l},\left(x_{0}\right)_{\alpha r}$ are bounded uniformly in $\alpha \in[0,1], \sigma_{\alpha l}(s), \sigma_{\alpha r}(s)$ are bounded on $[0, t]$ uniformly in $\alpha$ ( $\sigma$ is bounded in the compact $[0, t]$ by continuity). The same behavior can be checked for the left-sided quotients

$$
\left(\frac{I^{1-q} x(t)-I_{H} I^{1-q} x(t-h)}{h}\right)_{\alpha l}, \quad \text { and }\left(\frac{I^{1-q} x(t)-{ }_{H} I^{1-q} x(t-h)}{h}\right)_{\alpha r}, \quad h>0 .
$$

This proves that

$$
\lim _{h \rightarrow 0^{+}} d_{H}\left(\left[\left(\frac{I^{1-q} x(t+h)-I^{1-q} x(t)}{h}\right)\right]^{\alpha},\left[z_{\alpha l}(t), z_{\alpha r}(t)\right]\right)=0
$$

uniformly in $\alpha$ by Definition 2.4, so that

$$
\lim _{h \rightarrow 0^{+}} d_{\infty}\left(D^{q} x(t), z(t)\right)=0
$$

where for $t \in(0,+\infty),[z(t)]^{\alpha}=\left[z_{\alpha l}(t), z_{\alpha r}(t)\right]$ is a fuzzy number since $E^{1}$ is complete. Note that

$$
D^{q} x(t)+M x(t)=z(t)+M x(t)=\sigma(t), \quad t \in(0,+\infty),
$$

and $\lim _{t \rightarrow 0^{+}} t^{1-q} x(t)=x_{0}$, so that we obtain the solution of (3.1).

Theorem 3.2 If the nonnegative map

$$
t \rightarrow t^{1-q} \int_{0}^{t}(t-s)^{q-1} E_{q, q}\left(-M(t-s)^{q}\right) d_{\infty}\left(\sigma(s), \chi_{\{0\}}\right) d s, \quad t \in \mathbb{R}^{+}
$$

is bounded and $x$ is the unique solution of problem (3.1), then $t^{1-q} x$ is bounded. If $x_{0}=\chi_{\{0\}}$ and the nonnegative map

$$
t \rightarrow \int_{0}^{t}(t-s)^{q-1} E_{q, q}\left(-M(t-s)^{q}\right) d_{\infty}\left(\sigma(s), \chi_{\{0\}}\right) d s, \quad t \in \mathbb{R}^{+}
$$

is bounded, then $x$ is bounded. 
Proof For $t \in \mathbb{R}^{+}$, we have

$$
\begin{aligned}
d_{\infty}\left(t^{1-q} x(t), \chi_{\{0\}}\right) \leq & \Gamma(q) E_{q, q}\left(-M t^{q}\right) d_{\infty}\left(x_{0}, \chi_{\{0\}}\right) \\
& +t^{1-q} \int_{0}^{t}(t-s)^{q-1} E_{q, q}\left(-M(t-s)^{q}\right) d_{\infty}\left(\sigma(s), \chi_{\{0\}}\right) d s \\
\leq & d_{\infty}\left(x_{0}, \chi_{\{0\}}\right) \\
& +t^{1-q} \int_{0}^{t}(t-s)^{q-1} E_{q, q}\left(-M(t-s)^{q}\right) d_{\infty}\left(\sigma(s), \chi_{\{0\}}\right) d s
\end{aligned}
$$

which implies that $t^{1-q} x$ is bounded.

If $x_{0}=\chi_{\{0\}}$, we conclude that

$$
d_{\infty}\left(x(t), \chi_{\{0\}}\right) \leq \int_{0}^{t}(t-s)^{q-1} E_{q, q}\left(-M(t-s)^{q}\right) d_{\infty}\left(\sigma(s), \chi_{\{0\}}\right) d s
$$

and the proof is complete.

Corollary 3.1 If $t^{1-q} \sigma(t)$ is bounded, and $x$ is the unique solution of problem (3.1), then $t^{1-q} x$ is bounded. If $x_{0}=\chi_{\{0\}}$ and $\sigma$ is bounded, then $x$ is bounded.

Proof Firstly, it is easy to calculate that

$$
t^{2 q-1} E_{q, 2 q}\left(-M t^{q}\right)=\frac{t^{q-1}}{-M}\left(E_{q, q}\left(-M t^{q}\right)-\frac{1}{\Gamma(q)}\right) .
$$

Then it follows from Lemmas 2.3, 2.5 that

$$
\begin{aligned}
& t^{1-q} \int_{0}^{t}(t-s)^{q-1} E_{q, q}\left(-M(t-s)^{q}\right) d_{\infty}\left(\sigma(s), \chi_{\{0\}}\right) d s \\
& =t^{1-q} \int_{0}^{t}(t-s)^{q-1} E_{q, q}\left(-M(t-s)^{q}\right) s^{q-1} d_{\infty}\left(s^{1-q} \sigma(s), \chi_{\{0\}}\right) d s \\
& \quad \leq K t^{1-q} \int_{0}^{t}(t-s)^{q-1} E_{q, q}\left(-M(t-s)^{q}\right) s^{q-1} d s \\
& =K t^{1-q} \Gamma(q) t^{2 q-1} E_{q, 2 q}\left(-M t^{q}\right) \\
& =K \Gamma(q) \frac{1}{-M}\left(E_{q, q}\left(-M t^{q}\right)-\frac{1}{\Gamma(q)}\right) \leq \frac{K}{M} .
\end{aligned}
$$

Finally, by hypothesis, there exists $K \geq 0$ such that

$$
d_{\infty}\left(t^{1-q} \sigma(t), \chi_{\{0\}}\right) \leq K, \quad t \in \mathbb{R}^{+}
$$

Therefore, in this case, for $t \in \mathbb{R}^{+}$,

$$
d_{\infty}\left(t^{1-q} x(t), \chi_{\{0\}}\right) \leq \Gamma(q) E_{q, q}\left(-M t^{q}\right) d_{\infty}\left(x_{0}, \chi_{\{0\}}\right)+\frac{K}{M} \leq d_{\infty}\left(x_{0}, \chi_{\{0\}}\right)+\frac{K}{M} .
$$

In the case of $x_{0}=\chi_{\{0\}}$, if $\sigma$ is bounded, then there exists $K \geq 0$ such that

$$
d_{\infty}\left(\sigma(t), \chi_{\{0\}}\right) \leq K, \quad t \in \mathbb{R}^{+} .
$$


Hence, by Lemmas $2.3,2.4$, for $t \in \mathbb{R}^{+}$,

$$
\begin{aligned}
d_{\infty}\left(x(t), \chi_{\{0\}}\right) & \leq K \int_{0}^{t}(t-s)^{q-1} E_{q, q}\left(-M(t-s)^{q}\right) d s \\
& =K \frac{1-E_{q}\left(-M t^{q}\right)}{M} \leq \frac{K}{M} .
\end{aligned}
$$

The proof is therefore complete.

Example 3.1 Consider the following problem:

$$
\left\{\begin{array}{l}
D^{\frac{1}{2}} x(t)+x(t)=\sigma(t), \quad t \in(0,+\infty), \\
\lim _{t \rightarrow 0^{+}} t^{1-q} x(t)=\chi_{\{0\}},
\end{array}\right.
$$

where $q=\frac{1}{2}, M=1$ and $\sigma$ is a 0 -symmetric constant fuzzy number with level sets $[\sigma]^{\alpha}=$ $\left[-\frac{1}{1+\alpha}, \frac{1}{1+\alpha}\right]$ for every $\alpha \in[0,1]$.

Firstly, by (3.6) and Lemma 2.4, we have

$$
\begin{aligned}
\operatorname{diam}\left([x(t)]^{\alpha}\right) & =\int_{0}^{t} \operatorname{diam}\left([\sigma(s)]^{\alpha}\right)(t-s)^{-\frac{1}{2}} E_{\frac{1}{2}, \frac{1}{2}}\left(-(t-s)^{\frac{1}{2}}\right) d s \\
& =\frac{2}{1+\alpha}\left(1-E_{\frac{1}{2}}\left(-t^{\frac{1}{2}}\right)\right)
\end{aligned}
$$

is a nondecreasing function in $t$ for every $\alpha \in[0,1]$.

Secondly, using Lemmas 2.3 and 2.4 , we obtain

$$
\begin{aligned}
x(t+h)_{\alpha l}-x(t)_{\alpha l}= & \int_{0}^{t+h} \sigma_{\alpha l}(s)(t+h-s)^{-\frac{1}{2}} E_{\frac{1}{2}, \frac{1}{2}}\left(-(t+h-s)^{\frac{1}{2}}\right) d s \\
& -\int_{0}^{t} \sigma_{\alpha l}(s)(t-s)^{-\frac{1}{2}} E_{\frac{1}{2}, \frac{1}{2}}\left(-(t-s)^{\frac{1}{2}}\right) d s \\
= & -\frac{1}{1+\alpha}\left(E_{\frac{1}{2}}\left(-t^{\frac{1}{2}}\right)-E_{\frac{1}{2}}\left(-(t+h)^{\frac{1}{2}}\right)\right),
\end{aligned}
$$

which implies that $x(t+h)_{\alpha l}-x(t)_{\alpha l}$ is nondecreasing in $\alpha$. Similarly, we have $x(t)_{\alpha l}-x(t-$ $h)_{\alpha l}$ is a nondecreasing function in $\alpha$ and $x(t+h)_{\alpha r}-x(t)_{\alpha r}, x(t)_{\alpha r}-x(t-h)_{\alpha r}$ are nonincreasing functions in $\alpha$.

Hence, the $H$-differences $x(t+h)-{ }_{H} x(t)$ and $x(t)-{ }_{H} x(t-h)$ exist. By Corollary 3.1, it follows that the unique solution $x$ of problem (3.7) is bounded.

In fact, the solution of problem (3.7) can be solved by $[x(t)]^{\alpha}=\left[-\frac{1-E_{\frac{1}{2}}\left(-t^{\frac{1}{2}}\right)}{1+\alpha}, \frac{1-E_{\frac{1}{2}}\left(-t^{\frac{1}{2}}\right)}{1+\alpha}\right]$, which is represented for $t \in[0,20]$ in Figure 1.

\section{Main results}

Lemma 4.1 ([19]) Let $m, h, q, v \in C\left(\mathbb{R}^{+}, \mathbb{R}^{+}\right)$and suppose that

$$
m(t) \leq h(t)+\int_{t_{0}}^{t} q(t) v(s) m(s) d s, \quad t \geq t_{0}
$$




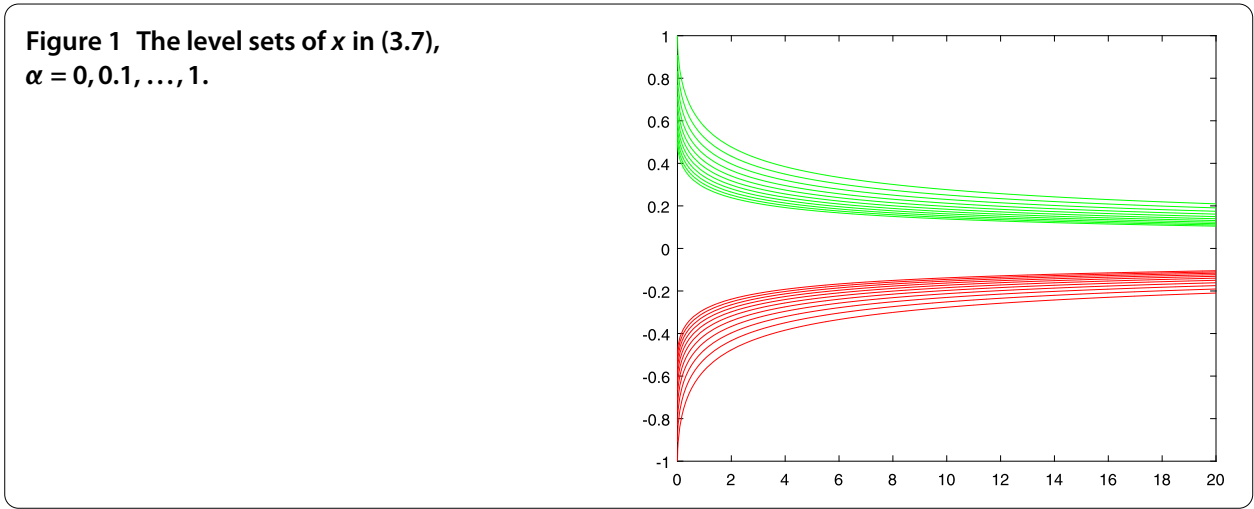

Then

$$
m(t) \leq h(t)+q(t) \int_{t_{0}}^{t} e^{\int_{s}^{t} v(\xi) q(\xi) d \xi} v(s) h(s) d s, \quad t \geq t_{0} .
$$

Theorem 4.1 Let $m, v_{1}, v_{2}, q_{1}, q_{2}, h \in C\left(\mathbb{R}^{+}, \mathbb{R}^{+}\right)$such that

$$
m(t) \leq h(t)+\int_{0}^{t} q_{1}(t) v_{1}(s) m(s) d s+\int_{0}^{t} q_{2}(t) v_{2}(s) \sup _{0 \leq \theta \leq s} m(\theta) d s, \quad t \in \mathbb{R}^{+}
$$

Then

$$
m(t) \leq\left(1+\sup _{0 \leq s \leq t} h(s)\right) e^{\sup _{0 \leq r \leq t} Q(r) \int_{0}^{t} V(\xi) d \xi}, \quad t \in \mathbb{R}^{+},
$$

where $V(t)=v_{1}(t)+v_{2}(t)$ and $Q(t)=\sup \left\{q_{1}(t), q_{2}(t)\right\}$.

Proof Let

$$
x(t)= \begin{cases}1, & 0 \leq m(t) \leq 1, \\ m(t), & m(t)>1\end{cases}
$$

Then, for $t \in \mathbb{R}^{+}$, we have $\max \{1, m(t)\} \leq x(t) \leq 1+m(t)$, and hence by (4.1)

$$
\begin{aligned}
x(t) & \leq 1+h(t)+\int_{0}^{t} q_{1}(t) v_{1}(s) x(s) d s+\int_{0}^{t} q_{2}(t) v_{2}(s) \sup _{0 \leq \theta \leq s} x(\theta) d s \\
& \leq 1+h(t)+Q(t) \int_{0}^{t} V(\tau) \sup _{0 \leq \theta \leq \tau} x(\theta) d \tau .
\end{aligned}
$$

Thus,

$$
\sup _{0 \leq s \leq t} x(s) \leq 1+\sup _{0 \leq s \leq t} h(s)+\sup _{0 \leq s \leq t} Q(s) \int_{0}^{t} V(\tau) \sup _{0 \leq \theta \leq \tau} x(\theta) d \tau .
$$


Together with Lemma 4.1, we can get

$$
\begin{aligned}
\sup _{0 \leq s \leq t} x(s) \leq & 1+\sup _{0 \leq s \leq t} h(s)+\sup _{0 \leq s \leq t} Q(s) \int_{0}^{t} e^{\int_{\tau}^{t} V(\xi) \sup _{0 \leq r \leq \xi} Q(r) d \xi} \\
& \times V(\tau)\left(1+\sup _{0 \leq r \leq \tau} h(r)\right) d \tau \\
\leq & \left(1+\sup _{0 \leq s \leq t} h(s)\right)\left(1+\sup _{0 \leq s \leq t} Q(s) \int_{0}^{t} e^{\int_{\tau}^{t} V(\xi) \sup _{0 \leq r \leq \xi} Q(r) d \xi} V(\tau) d \tau\right) \\
\leq & \left(1+\sup _{0 \leq s \leq t} h(s)\right)\left(1+\sup _{0 \leq s \leq t} Q(s) \int_{0}^{t} e^{\sup _{0 \leq r \leq t} Q(r) \int_{\tau}^{t} V(\xi) d \xi} V(\tau) d \tau\right) \\
= & \left(1+\sup _{0 \leq s \leq t} h(s)\right) e^{\sup _{0 \leq r \leq t} Q(r) \int_{0}^{t} V(\xi) d \xi},
\end{aligned}
$$

where we used the fact that

$$
\sup _{0 \leq s \leq t} Q(s) \int_{0}^{t} e^{\sup _{0 \leq r \leq t} Q(r) \int_{\tau}^{t} V(\xi) d \xi} V(\tau) d \tau=e^{\sup _{0 \leq r \leq t} Q(r) \int_{0}^{t} V(\xi) d \xi}-1
$$

note that $m(t) \leq x(t) \leq \sup _{0 \leq s \leq t} x(s)$, we can complete the proof.

Remark 4.1 If $h$ in Theorem 4.1 is nondecreasing, $q_{1}(t)=q_{2}(t) \equiv 1$, we can obtain the estimate

$$
m(t) \leq[1+h(t)] e^{\int_{0}^{t} V(\xi) d \xi}
$$

Theorem 4.2 (Generalized Gronwall inequality) For $\beta_{i}, \gamma_{i} \in(0,1], i=1,2$, let $1<p<$ $\min \left\{\frac{1}{1-\beta_{i}}, \frac{1}{1-\gamma_{i}}: i=1,2\right\}$ and $m, h, v_{1}, v_{2}, t^{p\left(\beta_{1}+\gamma_{1}-2\right)+1} q_{1}^{p}, t^{p\left(\beta_{2}+\gamma_{2}-2\right)+1} q_{2}^{p} \in C\left(\mathbb{R}^{+}, \mathbb{R}^{+}\right)$such that

$$
\begin{aligned}
m(t) \leq & h(t)+\int_{0}^{t}(t-s)^{\beta_{1}-1} s^{\gamma_{1}-1} q_{1}(t) v_{1}(s) m(s) d s \\
& +\int_{0}^{t}(t-s)^{\beta_{2}-1} s^{\gamma_{2}-1} q_{2}(t) v_{2}(s) \sup _{0 \leq \theta \leq s} m(\theta) d s, \quad t \in \mathbb{R}^{+}
\end{aligned}
$$

Then

$$
m(t) \leq\left[\left(1+3^{\frac{1}{p-1}} \sup _{0 \leq s \leq t} h^{\frac{p}{p-1}}(s)\right) e^{3^{\frac{1}{p-1}} \sup _{0 \leq r \leq t} Q(r) \int_{0}^{t} V(\xi) d \xi}\right]^{\frac{p-1}{p}}, \quad t \in \mathbb{R}^{+}
$$

where $V(t)=v_{1}^{\frac{p}{p-1}}(t)+v_{2}^{\frac{p}{p-1}}(t)$ and

$$
Q(t)=\sup \left\{q_{i}^{\frac{p}{p-1}}(t)\left[B\left(p\left(\gamma_{i}-1\right)+1, p\left(\beta_{i}-1\right)+1\right) t^{p\left(\beta_{i}+\gamma_{i}-2\right)+1}\right]^{\frac{1}{p-1}}, i=1,2\right\} .
$$

Proof We know that $\int_{0}^{t}(t-s)^{p(\beta-1)} s^{p(\gamma-1)} d s=B(p(\gamma-1)+1, p(\beta-1)+1) t^{p(\beta+\gamma-2)+1}$. Here, $B(\cdot, \cdot)$ denotes the beta function. 
It follows from condition (4.5) and the Hölder inequality that

$$
\begin{aligned}
m(t) \leq & h(t)+\int_{0}^{t}(t-s)^{\beta_{1}-1} s^{\gamma_{1}-1} q_{1}(t) v_{1}(s) m(s) d s \\
& +\int_{0}^{t}(t-s)^{\beta_{2}-1} s^{\gamma_{2}-1} q_{2}(t) v_{2}(s) \sup _{0 \leq \theta \leq s} m(\theta) d s \\
\leq & h(t)+q_{1}(t)\left[\int_{0}^{t}(t-s)^{p\left(\beta_{1}-1\right)} s^{p\left(\gamma_{1}-1\right)} d s\right]^{\frac{1}{p}}\left[\int_{0}^{t}\left(v_{1}(s) m(s)\right)^{\frac{p}{p-1}} d s\right]^{\frac{p-1}{p}} \\
& +q_{2}(t)\left[\int_{0}^{t}(t-s)^{p\left(\beta_{2}-1\right)} s^{p\left(\gamma_{2}-1\right)} d s\right]^{\frac{1}{p}}\left[\int_{0}^{t}\left(v_{2}(s) \sup _{0 \leq \theta \leq s} m(\theta)\right)^{\frac{p}{p-1}} d s\right]^{\frac{p-1}{p}} .
\end{aligned}
$$

Note that $\left(\sup _{0 \leq s \leq t} m(s)\right)^{\frac{p}{p-1}}=\sup _{0 \leq s \leq t} m^{\frac{p}{p-1}}(s)$; consequently,

$$
\begin{aligned}
m^{\frac{p}{p-1}}(t) \leq & 3^{\frac{1}{p-1}} h^{\frac{p}{p-1}}(t)+3^{\frac{1}{p-1}} Q(t) \int_{0}^{t}\left(v_{1}(s) m(s)\right)^{\frac{p}{p-1}} d s \\
& +3^{\frac{1}{p-1}} Q(t) \int_{0}^{t}\left(v_{2}(s) \sup _{0 \leq \theta \leq s} m(\theta)\right)^{\frac{p}{p-1}} d s .
\end{aligned}
$$

By Theorem 4.1, one can see that

$$
m^{\frac{p}{p-1}}(t) \leq\left(1+3^{\frac{1}{p-1}} \sup _{0 \leq s \leq t} h^{\frac{p}{p-1}}(s)\right) e^{\frac{1}{3^{p-1}} \sup _{0 \leq r \leq t} Q(r) \int_{0}^{t} V(\xi) d \xi},
$$

then we can complete the rest of the proof immediately.

Corollary 4.1 For $\beta, \gamma \in(0,1]$, let $1<p<\min \left\{\frac{1}{1-\beta}, \frac{1}{2-\gamma-\beta}\right\}$ and $m, h \in C_{1-\beta}\left(\mathbb{R}^{+}, \mathbb{R}^{+}\right), v$, $t^{p(\gamma+\beta-2)+1} q^{p} \in C\left(\mathbb{R}^{+}, \mathbb{R}^{+}\right)$such that

$$
m(t) \leq h(t)+\int_{0}^{t}(t-s)^{\beta-1} s^{\gamma-1} q(t) v(s) m(s) d s, \quad t \in(0,+\infty) .
$$

Then, for $t \in(0,+\infty)$,

$$
t^{1-\beta} m(t) \leq\left[\left(1+3^{\frac{1}{p-1}} \sup _{0 \leq s \leq t}\left(s^{1-\beta} h(s)\right)^{\frac{p}{p-1}}\right) e^{\frac{1}{p-1} \sup _{0 \leq r \leq t} Q(r) \int_{0}^{t} V(\xi) d \xi}\right]^{\frac{p-1}{p}}
$$

where $V(t)=v^{\frac{p}{p-1}}(t)$ and

$$
Q(t)=\left(t^{1-\beta} q(t)\right)^{\frac{p}{p-1}}\left[B(p(\gamma+\beta-2)+1, p(\beta-1)+1) t^{p(\gamma+2 \beta-3)+1}\right]^{\frac{1}{p-1}} .
$$

Proof Using (4.8), we obtain

$$
t^{1-\beta} m(t) \leq t^{1-\beta} h(t)+t^{1-\beta} q(t) \int_{0}^{t}(t-s)^{\beta-1} s^{\gamma+\beta-2} v(s) s^{1-\beta} m(s) d s, \quad t \in(0,+\infty),
$$

then the conclusion follows from Theorem 4.2.

We now apply the previous results to obtain bounds for the solutions of fuzzy fractional integral equations. 
Theorem 4.3 Let $1<p<\frac{1}{1-q}$ and $f \in C\left(\mathbb{R}^{+}, E^{n}\right)$ be bounded and

$$
G_{i}: \Delta=\{(t, s): 0 \leq s \leq t<+\infty\} \rightarrow \mathbb{R}
$$

be continuous such that

$$
\left|G_{i}(t, s)\right| \leq w_{i}(t) v_{i}(s), \quad \forall(t, s) \in \Delta, i=1,2,
$$

where $t^{p(q-1)+1} w_{i}^{p}, v_{i} \in C\left(\mathbb{R}^{+}, \mathbb{R}^{+}\right)$. Assume that the map

$$
t \rightarrow \sup _{0 \leq r \leq t} Q(r) \int_{0}^{t} V(\xi) d \xi, \quad t \in \mathbb{R}^{+} \text {is bounded }
$$

where $V(t)=v_{1}^{\frac{p}{p-1}}(t)+v_{2}^{\frac{p}{p-1}}(t)$ and

$$
Q(t)=\sup \left\{w_{i}^{\frac{p}{p-1}}(t)\left[B(1, p(q-1)+1) t^{p(q-1)+1}\right]^{\frac{1}{p-1}}: i=1,2\right\}
$$

Then all the solutions of fuzzy fractional functional integral equation (1.3) are bounded.

Proof Let $x$ be a solution of (1.3), and let $m(t)=d_{\infty}\left(x(t), \chi_{\{0\}}\right), t \in \mathbb{R}^{+}$, then

$$
\begin{aligned}
m(t)= & d_{\infty}\left(\int_{0}^{t}(t-s)^{q-1}\left[G_{1}(t, s) x(s)+G_{2}(t, s) x(\theta(s))\right] d s+f(t), \chi_{\{0\}}\right) \\
\leq & d_{\infty}\left(\int_{0}^{t}(t-s)^{q-1} G_{1}(t, s) x(s) d s, \chi_{\{0\}}\right) \\
& +d_{\infty}\left(\int_{0}^{t}(t-s)^{q-1} G_{2}(t, s) x(\theta(s)) d s, \chi_{\{0\}}\right)+d_{\infty}\left(f(t), \chi_{\{0\}}\right) \\
\leq & \int_{0}^{t}(t-s)^{q-1}\left|G_{1}(t, s)\right| m(s) d s \\
& +\int_{0}^{t}(t-s)^{q-1}\left|G_{2}(t, s)\right| m(\theta(s)) d s+d_{\infty}\left(f(t), \chi_{\{0\}}\right) \\
\leq & w_{1}(t) \int_{0}^{t}(t-s)^{q-1} v_{1}(s) m(s) d s \\
& +w_{2}(t) \int_{0}^{t}(t-s)^{q-1} v_{2}(s) m(\theta(s)) d s+d_{\infty}\left(f(t), \chi_{\{0\}}\right), \quad t \in \mathbb{R}^{+} .
\end{aligned}
$$

Applying Theorem 4.2, we get

$$
m(t) \leq\left[\left(1+3^{\frac{1}{p-1}} \sup _{0 \leq s \leq t} d_{\infty}^{\frac{p}{p-1}}\left(f(s), \chi_{\{0\}}\right)\right) e^{3^{\frac{1}{p-1}} \sup _{0 \leq r \leq t} Q(r) \int_{0}^{t} V(\xi) d \xi}\right]^{\frac{p-1}{p}}
$$

and the proof is complete.

Example 4.1 Let us consider the fuzzy fractional order functional integral equation

$$
x(t)=\int_{0}^{t}(t-s)^{-0.25} \frac{1}{e^{s} \sqrt{1+t}} x(\theta(s)) d s+f(t)
$$


where $f: \mathbb{R}^{+} \rightarrow E^{1}$ is defined by the $\alpha$-cut $[f(t)]^{\alpha}=\left[\frac{\alpha}{1+t}, \frac{2-\alpha}{1+t}\right], 0 \leq \theta(t) \leq t, p=2, q=$ $0.75, G_{1}(t, s)=0, G_{2}(t, s)=\frac{1}{e^{5} \sqrt{1+t}}$. Observe that $d_{\infty}\left(f(t), \chi_{\{0\}}\right)=\sup _{\alpha \in[0,1]} \max \left\{\frac{\alpha}{1+t}, \frac{2-\alpha}{1+t}\right\} \leq 1$, which implies that $f$ is bounded.

Let $w(t)=\frac{1}{\sqrt{1+t}}, v(t)=e^{-t}$, then $V(t)=e^{-2 t}$ and $Q(t)=\frac{\sqrt{t}}{1+t} B(1,0.5)$. Obviously, $\sup _{0 \leq r \leq t} Q(r) \int_{0}^{t} V(\xi) d \xi \leq \frac{B(1,0.5)}{2}$. Now, conditions in Theorem 4.3 are satisfied, which guarantees all the solutions of this fuzzy fractional functional integral equation are bounded.

Theorem 4.4 Let $1<p<\frac{1}{1-q}, f \in C_{1-q}\left(\mathbb{R}^{+}, E^{n}\right)$ and $t^{1-q} f$ be bounded and

$$
G: \Delta=\{(t, s): 0 \leq s \leq t<+\infty\} \rightarrow \mathbb{R}
$$

be continuous such that

$$
|G(t, s)| \leq w(t) v(s), \quad \forall(t, s) \in \Delta,
$$

where $t^{p(q-1)+1} w^{p}, v \in C\left(\mathbb{R}^{+}, \mathbb{R}^{+}\right)$. Assume that the map

$$
t \rightarrow \sup _{0 \leq r \leq t} Q(r) \int_{0}^{t} V(\xi) d \xi, \quad t \in \mathbb{R}^{+} \text {is bounded, }
$$

where $V(t)=v^{\frac{p}{p-1}}(t)$ and

$$
Q(t)=\left(t^{1-q} w(t)\right)^{\frac{p}{p-1}}\left[B(p(q-1)+1, p(q-1)+1) t^{2 p(q-1)+1}\right]^{\frac{1}{p-1}} .
$$

Then, for all solutions $x$ of (1.4), $t^{1-q} x$ are bounded.

Proof If $x$ is a solution of (1.4) and $m(t)=d_{\infty}\left(x(t), \chi_{\{0\}}\right), t \in(0,+\infty)$, then

$$
m(t) \leq w(t) \int_{0}^{t}(t-s)^{q-1} v(s) m(s) d s+d_{\infty}\left(f(t), \chi_{\{0\}}\right), \quad t \in(0,+\infty) .
$$

Thus, the conditions of Corollary 4.1 are satisfied, and hence

$$
t^{1-q} m(t) \leq\left[\left(1+3^{\frac{1}{p-1}} \sup _{0 \leq s \leq t}\left(s^{1-q} d_{\infty}\left(f(s), \chi_{\{0\}}\right)\right)^{\frac{p}{p-1}}\right) e^{3^{\frac{1}{p-1}} \sup _{0 \leq r \leq t} Q(r) \int_{0}^{t} V(\xi) d \xi}\right]^{\frac{p-1}{p}}
$$

This completes the proof.

Example 4.2 Consider the following fractional order fuzzy integral equation:

$$
x(t)=\int_{0}^{t}(t-s)^{\frac{2}{3}} \frac{1}{1+t^{\frac{2}{3}}} x(s) d s+f(t)
$$

where $f \in C_{\frac{1}{3}}\left(\mathbb{R}^{+}, E^{1}\right)$ is given by the $\alpha$-cut $[f(t)]^{\alpha}=\left[t^{-\frac{1}{3}}(\alpha-1), t^{-\frac{1}{3}}(1-\alpha)\right], p=\frac{5}{4}, q=\frac{2}{3}$ and $G(t, s)=\frac{s}{1+t^{\frac{2}{3}}}$. It is easy to see that $t^{\frac{1}{3}} f$ is bounded. Moreover, $w(t)=\frac{1}{1+t^{\frac{2}{3}}}, v(t)=1, Q(t)=$ $\frac{t^{\frac{7}{3}}}{\left(1+t^{\frac{2}{3}}\right)^{5}} B^{5}\left(\frac{7}{12}, \frac{7}{12}\right)$ and $\sup _{0 \leq r \leq t} Q(r) \int_{0}^{t} V(\xi) d \xi \leq B^{5}\left(\frac{7}{12}, \frac{7}{12}\right)$. Theorem 4.4 implies that, for all solutions $x$ of this fuzzy fractional integral equation, $t^{\frac{1}{3}} x$ are bounded. 


\section{Conclusions}

In the present paper, we consider the boundedness of solutions of certain fractional fuzzy differential equations as well as fuzzy integral equations. By introducing some differential and integral inequalities, which are more general than those in the previous literature, we obtain some results for the boundedness of solutions of fractional fuzzy integral equations.

\section{Acknowledgements}

The author is grateful to the editor and the anonymous reviewers for their constructive comments and suggestions which improved the quality of the paper.

\section{Funding}

This research was funded by Research Fund of Hunan Provincial Education Department (15C0538).

\section{Abbreviations}

Not applicable.

\section{Availability of data and materials}

Not applicable.

\section{Ethics approval and consent to participate}

Not applicable.

\section{Competing interests}

The author declares that he has no competing interests.

Consent for publication

Not applicable.

\section{Authors' contributions}

The author contributed to the writing of this paper and approved the final manuscript.

\section{Publisher's Note}

Springer Nature remains neutral with regard to jurisdictional claims in published maps and institutional affiliations.

Received: 4 July 2017 Accepted: 15 January 2018 Published online: 29 January 2018

\section{References}

1. Chang, SSL, Zadeh, LA: On fuzzy mapping and control. IEEE Trans. Syst. Man Cybern. 2, 30-34 (1972)

2. Kaleva, O: Fuzzy differential equations. Fuzzy Sets Syst. 24, 301-317 (1987)

3. Puri, ML, Ralescu, DA: Differentials for fuzzy functions. J. Math. Anal. Appl. 91, 552-558 (1983)

4. Dubois, D, Prade, H: Towards fuzzy differential calculus. I. Integration of fuzzy mappings. Fuzzy Sets Syst. 8, 1-17 (1982)

5. Kaleva, O: The Cauchy problem for fuzzy differential equations. Fuzzy Sets Syst. 35, 389-396 (1990)

6. Park, JY, Jeong, JU: A note on fuzzy integral equations. Fuzzy Sets Syst. 108, 193-200 (1999)

7. O'Regan, D, Lakshmikantham, V, Nieto, JJ: Initial and boundary value problems for fuzzy differential equations. Nonlinear Anal. 54, 405-415 (2003)

8. Seikkala, S: On the fuzzy initial value problem. Fuzzy Sets Syst. 24, 319-330 (1987)

9. Nieto, JJ, Rodríguez-López, R: Bounded solutions for fuzzy differential and integral equations. Chaos Solitons Fractals 27, 1376-1386 (2006)

10. Georgiou, DN, Kougias, IE: Bounded solutions for fuzzy integral equations. Int. J. Math. Math. Sci. 31, 109-114 (2002)

11. Kilbas, AA, Srivastava, HM, Trujillo, Jj: Theory and Applications of Fractional Differential Equations. North-Holland Mathematics Studies, vol. 204. Elsevier, Amsterdam (2006)

12. Agarwal, RP, Arshad, $S, O^{\prime}$ Regan, $D$, Lupulescu, V: Fuzzy fractional integral equations under compactness type condition. Fract. Calc. Appl. Anal. 15, 572-590 (2012)

13. Hale, JK: Asymptotic Behavior of Dissipative Systems, vol. 25. Am. Math. Soc., Providence (1988)

14. Lakshmikantham, V, Vatsala, AS: Theory of fractional differential inequalities and applications. Commun. Appl. Anal. $11,871-879(2007)$

15. Lakshmikantham, V, Leela, S: Differential and Integral Inequalities. Academic Press, New York (1969)

16. Wang, JR, Fečkan, M, Zhou, Y: Presentation of solutions of impulsive fractional Langevin equations and existence results. Eur. Phys. J. Spec. Top. 222, 1857-1874 (2013)

17. Prabhakar, TR: A singular integral equation with a generalized Mittag-Leffler function in the kernel. Yokohama Math. J. 19,7-15 (1971)

18. Lakshmikantham, V, Mohapatra, RN: Theory of Fuzzy Differential Equations and Inclusions. Taylor \& Francis, London (2003)

19. Lakshmikantham, V, Leela, S, Martynyuk, AA: Stability Analysis of Nonlinear Systems. Dekker, New York (1989) 